\title{
Constraints On Additional Z Bosons Derived From Neutrino-Electron Scattering Measurements
}

\section{CHARM II Collaboration}

\author{
P. Vilain ${ }^{1)}$, G. Wilquet ${ }^{1)}$ \\ Inter-University Institute for High Energies (ULB-VUB), Brussels, Belgium
}

R. Beyer ${ }^{2)}$, W. Flegel, H. Grote, T. Mouthuy ${ }^{3)}$, H. Øverås, J. Panman, A. Rozanov ${ }^{4}$, K. Winter, G. Zacek ${ }^{5}$, V. Zacek ${ }^{5}$ )

CERN, Geneva, Switzerland

F. W. Büsser, C. Foos, L. Gerland, T. Layda ${ }^{6}$, F. Niebergall, G. Rädel ${ }^{2)}$, P. Stähelin,

T. Voss

II. Institut für Experimentalphysik ${ }^{7)}$, Universität Hamburg, Hamburg, Germany

D. Favart, G. Grégoire, E. Knoops ${ }^{8)}$, V. Lemaître

Université Catholique de Louvain, Louvain-la-Neuve, Belgium

P. Gorbunov, E. Grigoriev, V. Khovansky, A. Maslennikov

Institute for Theoretical and Experimental Physics, Moscow, Russian Federation

W. Lippich, A. Nathaniel, A. Staude, J. Vogt

Sektion Physik ${ }^{7)}$ der Universität Müchen, Munich, Germany

\begin{abstract}
A. G. Cocco, A. Ereditato, G. Fiorillo, F. Marchetti-Stasi, V. Palladino, P. Strolin
\end{abstract} Università 'Federico II' and Istituto Nazionale di Fisica Nucleare (INFN), Naples, Italy

A. Capone, D. De Pedis, U. Dore, A. Frenkel-Rambaldi, P. F. Loverre, D. Macina, G. Piredda, R. Santacesaria

Università 'La Sapienza' and Istituto Nazionale di Fisica Nucleare (INFN), Rome, Italy

E. Di Capua, S. Ricciardi, B. Saitta

Università di Ferrara and Istituto Nationale di Fisica Nucleare (INFN), Ferrara, Italy

B. Akkus, E. Arik, M. Serin-Zeyrek, R. Sever, P. Tolun

High Energy Physics Research Centre, YEFAM, Ankara, Turkey

K. Hiller, R. Nahnhauer, H. E. Roloff

$D E S Y$ - Institut für Hochenergiephysik, Zeuthen, Germany

\footnotetext{
Abstract

We report new constraints on the existence of additional $Z$ bosons derived from neutrino-electron scattering data. The analysis is based on data taken by the CHARM II Collaboration during five years of exposure of the detector to the wide band neutrino beam at CERN. The limits on the mass of these new particles are evaluated in the framework of $E_{6}$ superstring-inspired theories and Left-Right symmetric models. Limits on the so-called $Z^{1}$ are also reported.

1) National Foundation for Scientific Research, Belgium.

2) Now at DESY, Hamburg, Germany.

3) Now at Centre de Physique des Particules de Marseille, Faculté de Luminy, Marseille, France.

4) On leave of absence from ITEP, Moscow, Russian Federation.

5) Now at University of Montreal, Canada.

6) Now at University of California at Santa Cruz, USA.

7) Supported by the German Bundesministerium für Forschung und Technologie (BMFT), under contract numbers 05-5HH22P and $05-5 \mathrm{MU} 12 \mathrm{P}$.

8) Inter-University Institute for Nuclear Science, Belgium.
} 


\section{Introduction}

The existence of an additional $Z$ boson is foreseen in many extensions of the Standard Model of electroweak interactions. In this context, the analysis of weak neutral current data is of particular interest if this new particle is associated with an extra $U(1)$ symmetry, and has a mass in the range of 0.1 to $10 \mathrm{Tev}$.

Low-energy neutral current amplitudes are infact modified in the presence of an extra $Z$ boson mainly by the $Z^{\prime}$ exchange term, the $Z-Z^{\prime}$ mixing term, and to a lesser extent by the downward shift of the ordinary $Z$ mass. In particular, the vector coupling to charged leptons, which is usually small in the Standard Model, is very sensitive to the presence of an additional $Z$.

The high precision reached by the CHARM II experiment in the measurement of electroweak parameters [1] enables us to set new limits on the mass of this $Z^{\prime}$ boson. The outcome of this analysis together with the latest published results constrains further physics beyond the Standard Model.

\section{Theoretical framework}

Following the procedure described in Refs. [2, 3] in this paper we consider the limits that can be set on additional $Z$ bosons expected in grand unified theories (GUT), based on the $E_{6}$ gauge group, and on the Left-Right symmetric extension of the Standard Model. It is impractical, in fact, to deal with a completely general extended neutral current sector because, in addition to their masses and mixings, one must specify the couplings of each additional $Z$ to all relevant chiral fermions.

We will also discuss the limits on a heavy $Z^{\prime}$ boson with the same coupling as the ordinary $Z$, although such a particle is not expected in gauge theories.

The interest in the gauge group $E_{6}$ has been renewed by the possibility that a superstring theory might be a candidate for GUT [4]. In $E_{6}$ theories, additional gauge bosons can arise simply by the fact that the $E_{6}$ group has rank 6, two more than the Standard Model. As shown in Ref. [5], a possible scheme of the breakdown of this group is given by

$$
E_{6} \rightarrow S O(10) \times U(1)_{\psi},
$$

followed by

$$
S O(10) \rightarrow S U(5) \times U(1)_{\chi},
$$

where $S U(5)$ contains the standard $S U(3)_{C} \times$ $S U(2)_{L} \times U(1)_{Y}$. In this way if there is an additional neutral boson at low energy it can be expressed by a mixture of the two additional $U(1)$ groups present in the theory

$$
Z^{\prime}\left(\Theta_{E 6}\right)=Z_{\chi} \cos \Theta_{E 6}+Z_{\psi} \sin \Theta_{E 6}
$$

where $\Theta_{E 6}$ is a mixing angle, usually defined in the range $0 \leq \Theta_{E 6}<\pi$. The properties of this new neutral boson are thus a function of the $\Theta_{E 6}$ angle [2]. Values of $\Theta_{E 6}=0, \pi / 2$, and $\pi-\arctan \sqrt{5 / 3}$ define, respectively, the so-called $\chi, \psi$, and $\eta$ models $^{1}$.

With respect to Left-Right extensions of the Standard Model [6], the symmetry group $S U(2)_{L} \times$ $S U(2)_{R} \times U(1)_{L R}$ defines a light $Z_{L R}$, orthogonal to the ordinary $Z$, whose coupling strength to the ordinary fermions is parametrized [2] in terms of

$$
\alpha_{L R}=\sqrt{\frac{1-\left(1+g_{L}^{2} / g_{R}^{2}\right) \sin ^{2} \theta_{W}}{\left(g_{L}^{2} / g_{R}^{2}\right) \sin ^{2} \theta_{W}}},
$$

where $g_{L, R}$ are the $S U(2)_{L, R}$ coupling constants, and $\sin ^{2} \theta_{W}$ is the weak mixing angle. In particular, the Left-Right Symmetric (LR) model is characterized by $g_{L}=g_{R}$.

In each model the extra neutral boson $Z_{i}^{o}$ mixes with the ordinary $Z^{\circ}$ leading, after spontaneous symmetry breaking, to the two mass eigenstates

$$
\left(\begin{array}{c}
Z \\
Z_{i}^{\prime}
\end{array}\right)=\left(\begin{array}{rr}
\cos \theta & \sin \theta \\
-\sin \theta & \cos \theta
\end{array}\right)\left(\begin{array}{c}
Z^{\circ} \\
Z_{i}^{\circ}
\end{array}\right),
$$

where $i=\chi, \psi, \eta$, or $L R$. In this context the effective Lagrangian that describes the weak neutral current interaction becomes, in the presence of this additional boson,

$$
\mathcal{L}_{N C}=\frac{g}{\cos \theta_{W}} Z_{\mu}^{o}\left(J_{3}^{\mu}-\sin ^{2} \theta_{W} J_{e m}^{\mu}\right)+g^{\prime} Z_{i \mu}^{o} J_{i}^{\mu}
$$

where the index $i$ identifies the chosen model, as usual. This modified structure for the Lagrangian

\footnotetext{
${ }^{1}$ The $\psi$ and $\chi$ models occur when the first or, respectively, the second of the two sequential breakdowns dominate in the mixing, whilst the $Z_{\eta}$ boson occurs in many superstring-inspired models in which $E_{6}$ breaks directly into a rank-5 group.
} 
has the effect of changing the Standard Model couplings of the known fermions. In particular, for neutrino-electron scattering, the coupling constants $g_{V}^{e}$ and $g_{A}^{e}$ are replaced by $g_{V}^{\mathrm{eff}}$ and $g_{A}^{\mathrm{eff}}$, which are known functions of $\theta$ and $M_{Z^{\prime}}$ [2].

A precise measurement of $g_{V}$ and $g_{A}$ in neutrino-electron scattering could thus give interesting limits on possible physics beyond the Standard Model.

\section{Analysis procedure}

The CHARM II detector and its characteristics are described in detail in Refs. [7, 8]. The CHARM II Collaboration has recently published its final results [1] based on the complete sample of events collected during five years of exposure of the detector (from 1987 to 1991) to the wide band neutrino beam at CERN. The large statistics available, consisting of about 2670 neutrino-electron and 2750 antineutrino-electron scattering events yield the best determination of $g_{V}^{e}$ and $g_{A}^{e}$ in purely leptonic low- $Q^{2}$ neutral current interactions:

$$
g_{V}^{e}=-0.035 \pm 0.017 \quad g_{A}^{e}=-0.503 \pm 0.017 .
$$

Statistical and systematic errors have been added in quadrature.

The effective coupling constants derived from the complete neutral current amplitude in the presence of an additional $Z^{\prime}$ boson depends, as mentioned before, on the direct $Z$ and $Z^{\prime}$ exchange, and on their interference term. This dependence is parametrized in each model by $M_{Z_{i}}$ and the mixing angle $\theta$ :

$$
g_{V, A}^{\mathrm{eff}}=f_{V, A}^{i}\left(M_{o}, M_{Z_{i}}, \theta, \sin ^{2} \theta_{W}, m_{t}\right),
$$

where the index $i$ runs as usual on different models, and $M_{0}$ is the theoretical prediction for the $Z$ mass in the absence of mixing.

Couplings can thus obtain large contribution terms from the presence of an additional $Z$ boson; as an example of this, Fig. 1 shows $\delta g_{V}^{e}=$ $\left(g_{V}^{S M}-g_{V}^{\text {eff }}\right) / g_{V}^{S M}$ as a function of $M_{Z}$, in the $\chi$ model. Here $g_{V}^{S M}$ is the Standard Model prediction for the vector coupling of the electron in absence of additional $Z$.

Standard Model radiative corrections are applied to the tree-level expressions used to compute the effective couplings [9]; in accordance with

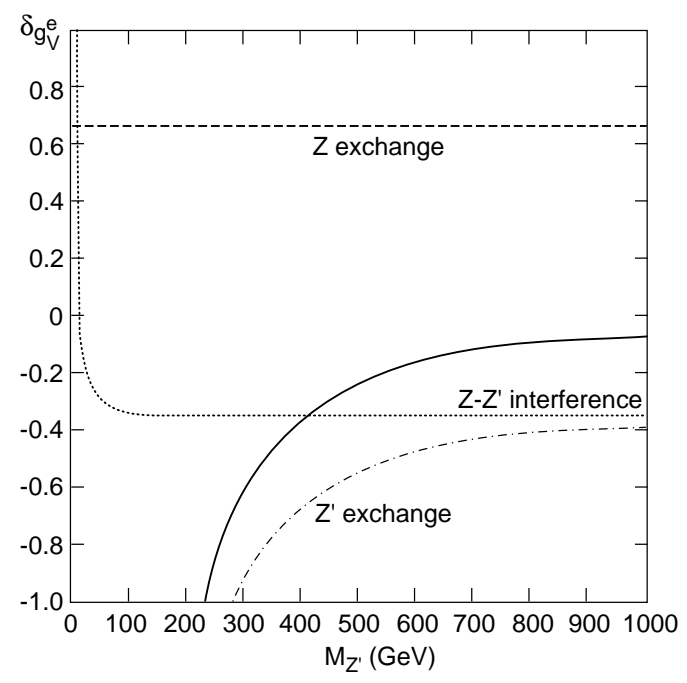

Figure 1: This figure shows $\delta g_{V}^{e}$ as a function of $M_{Z^{\prime}}$ in the $\chi$ model, assuming $\theta=0.001 \mathrm{rad}$ and $m_{t}=150 \mathrm{GeV}$. Also shown are the individual contributions of each of the three exchange terms.

Ref. [2], any new higher-order terms caused by $Z^{\prime}$ contribution have been neglected.

In order to reduce the number of parameters we have constrained the $Z$ mass to its measured value, $M_{Z}=91.187 \pm 0.007 \mathrm{GeV}[10]$. Using the relation that is valid for each model

$$
\tan ^{2} \theta=\frac{M_{o}^{2}-M_{Z}^{2}}{M_{Z_{i}^{\prime}}^{2}-M_{o}^{2}}
$$

and assuming that all $S U(2)$ breaking is due to Higgs doublets $(\rho=1)$ of known vacuum expectation values, leaves as free parameters only $M_{Z^{\prime}}, \theta$, and $m_{t}$.

For fixed values of $m_{t}$, the chi-squared variable

$$
\chi^{2}=\left(\frac{g_{V}^{\text {meas }}-g_{V}^{\text {eff }}}{\Delta g_{V}^{\text {meas }}}\right)^{2}+\left(\frac{g_{A}^{\text {meas }}-g_{A}^{\text {eff }}}{\Delta g_{A}^{\text {meas }}}\right)^{2},
$$

obtained by comparing the measured quantities and the expected ones is evaluated in the $\left(M_{Z^{\prime}}, \theta\right)$ plane; the limits on $M_{Z}$, and $\theta$ are given as exclusion plots.

In Fig. 2 we show the $95 \%$ confidence limits we derived, compared with the results of a recent analysis from L3 [11] that used the same parametrization. Assuming zero mixing the corresponding 

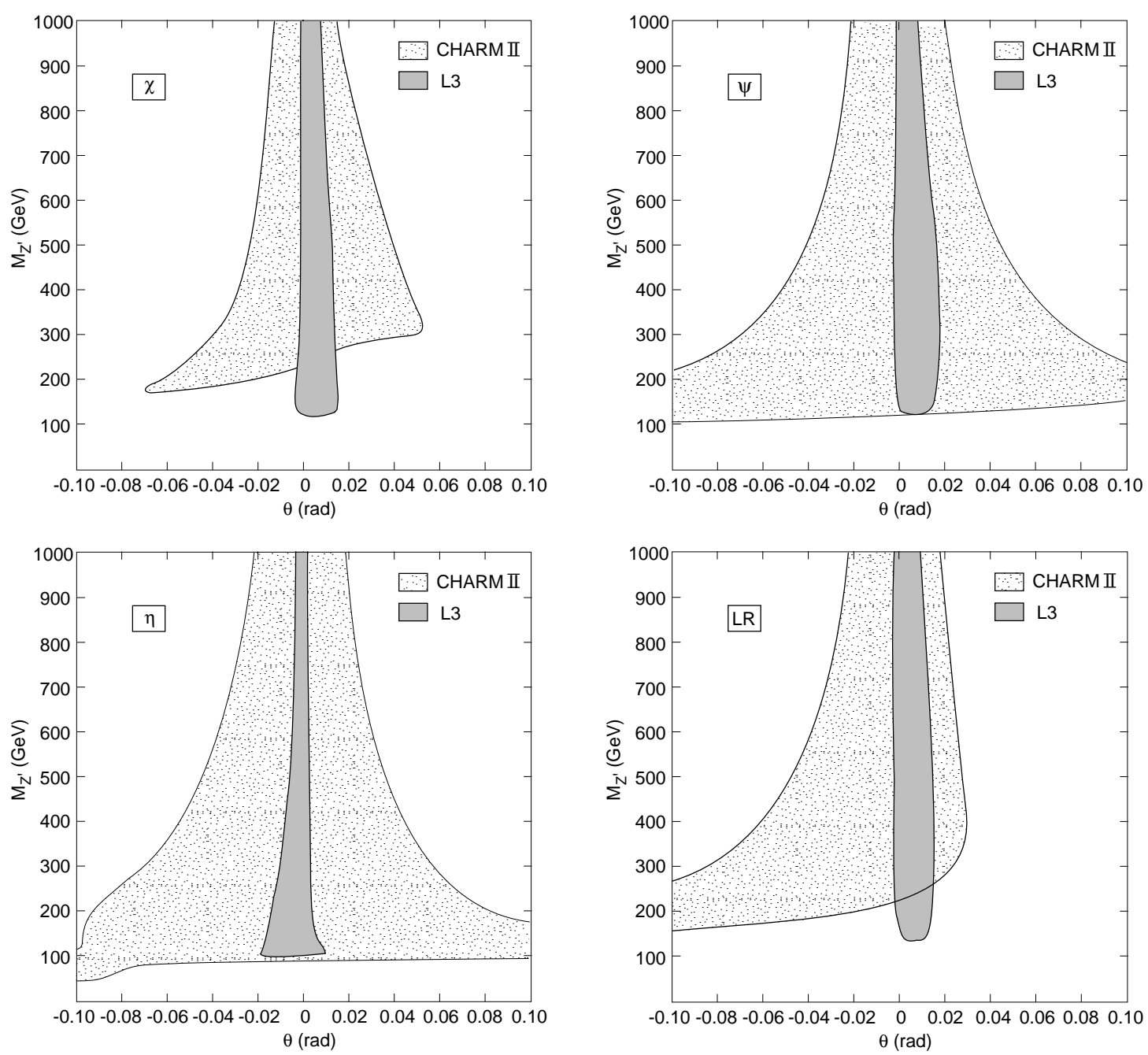

Figure 2: Contours in the $\theta-M_{Z}$, plane assuming $m_{t}=150 \mathrm{GeV}$. Values outside the shaded areas are excluded at the $95 \%$ CL. L3 contours were derived assuming $m_{H}=300 \mathrm{GeV}$ and $\alpha_{s}=0.12$.

mass limits are summarized in Table 1 for all the models.

In spite of the lower accuracy in the measurement of the coupling constants with respect to LEP experiments, the limits on $M_{Z^{\prime}}$ are typically better than those coming from quantities measured at the $Z^{\circ}$ peak; this is mainly due to the fact that low- $Q^{2}$ neutral current amplitudes are in principle more sensitive to the $Z-Z^{\prime}$ interference and $Z^{\prime}$ exchange term contributions. On the other hand, limits on $\theta$ are related to the downward shift of the $M_{Z}$ mass, i.e. to the total error on $g_{V}^{e}$ and $g_{A}^{e}[1,10]$ through Eq. (1).

For comparison, Table 1 also shows the best limits [12] on $Z^{\prime}$ mass which come from direct searches.
We now consider the limits that can be set on the so-called $Z^{1}$, a clone of the standard $Z$ not present in any gauge model, but useful to parametrize limits on new physics.

This neutral boson couples to the known fermions with the same strength as the ordinary $Z$, but has a larger mass. From the expression of the neutral current Lagrangian, and following the procedure described in [13], the Standard Model prediction for electron coupling constants to the neutral current are modified by

$$
g_{V, A}^{\mathrm{eff}}=g_{V, A}^{S M}+\Delta g_{V, A},
$$


Table 1: $95 \%$ lower limits for $M_{Z^{\prime}}$ (in GeV), assuming $m_{t}=150 \mathrm{GeV}$ and $\theta=0$. L3 bounds were derived by fixing $m_{H}=300 \mathrm{GeV}$ and $\alpha_{s}=$ 0.12. Also shown are the direct search limits from CDF, assuming $Z^{\prime}$ decay into known fermions (highest values) and all known and supersymmetric fermions (lowest values).

\begin{tabular}{ccc|c}
\hline Model & CHARM II & L3 & CDF \\
\hline$\chi$ & 262 & 117 & $280-340$ \\
$\psi$ & 135 & 118 & $180-320$ \\
$\eta$ & 100 & 100 & $230-340$ \\
LR & 253 & 130 & $230-310$ \\
\hline
\end{tabular}

where

$$
\Delta g_{V}=\frac{3}{5}\left(\frac{M_{Z}}{M_{Z^{1}}} \frac{g^{\prime}}{g}\right)^{2} \quad \Delta g_{A}=\frac{3}{10}\left(\frac{M_{Z}}{M_{Z^{1}}} \frac{g^{\prime}}{g}\right)^{2},
$$

and the gauge couplings ratio $g / g^{\prime}$ is taken to be equal to one.

To be compatible with the values measured by the CHARM II experiment the $Z^{1}$ must have a mass greater than

$$
M_{Z^{1}}>433 \mathrm{GeV} 90 \% \mathrm{CL} .
$$

This limit significantly improves the indirect bounds on the $Z^{1}$ mass derived from previous searches in the neutrino-electron scattering sector [14], it is competitive with the limits obtained from neutral current data analysis $[15,16]$ as shown by comparison of the CHARM II results with the latest Particle Data Group compilation given in Table $2[17]$.

Table 2: Indirect search limits on $M_{Z}$ at $90 \% \mathrm{CL}$. CHARM II results assume $m_{t}=150 \mathrm{GeV}$ and $\theta=0$

\begin{tabular}{cccccc}
\hline & $\chi$ & $\psi$ & $\eta$ & LR & $Z^{1}$ \\
\hline CHARM II & 287 & 150 & 108 & 278 & 433 \\
PDG & 320 & 154 & 125 & 325 & 426 \\
\hline
\end{tabular}

Moreover, when expressed at the $95 \% \mathrm{CL}$ $\left(M_{Z^{1}}>398 \mathrm{GeV}\right)$, the result of this analysis is comparable with the limits coming from the CDF direct search: $M_{Z^{1}}>412 \mathrm{GeV}$ [12].

\section{Conclusions}

The high-precision measurement of the electroweak parameters in the framework of the Standard Model enabled the CHARM II Collaboration to improve existing limits on the existence of extra neutral bosons coming from indirect searches in the weak neutral current sector; bounds on the mass of this boson are obtained in the most common theoretical models in the permitted region of mixing with the standard $Z^{\circ}$.

The limits obtained are competitive when compared with those from the direct search.

\section{Acknowledgements}

We gratefully acknowledge the skill and dedication of our numerous technical collaborators who have contributed to the realization and the operation of the detector and its associated systems. The experiment was made possible by grants from the Inter-University Institute for Nuclear Science (Belgium), CERN (Geneva, Switzerland), the Bundesministerium für Forschung und Technologie (Germany), the Institute of Theoretical and Experimental Physics (Moscow, Russian Federation), the Istituto Nazionale di Fisica Nucleare (Italy), and the Turkish Scientific and Technical Research Council (TUBITAK); we gratefully acknowledge their support. We thank the neutrino beam staff for their competent assistance ensuring the excellent performance of their facility. The accumulation of a large data sample in this experiment was made possible thanks to the skilful crew operating the CERN Super Proton Synchrotron.

We are indebted to G. Altarelli for useful discussions.

\section{References}

[1] CHARM II Collaboration, P. Vilain et al., Precision measurement of Electroweak parameters in neutrino-electron scattering, to be published in Physics Letters B.

A. Rozanov, Proceedings of the International Conference "NC Twenty Years Later" (Paris 1993).

R. Beyer, Proceedings of the EPS-HEP Conference (Marseille, 1993). 
[2] P. Langacker and M.Luo, Phys. Rev. D45 (1992) 278 .

[3] V.A. Bednyakov and S.G. Kovalenko, JINR/E2-88-395 (1988).

[4] P. Langacker and M. Luo, Phys. Rev. D44 (1991) 817, and references therein.

[5] D. London and J.L. Rosner, Phys. Rev. D34 (1986) 1530, and references therein.

[6] R.W. Robinett and J.L. Rosner, Phys. Rev. D25 (1982) 3036.

C.N. Leung and J.L. Rosner, Phys. Rev. D29 (1982) 2132, and references therein.

[7] CHARM II Collaboration, K. De Winter et al., Nucl. Instrum. and Methods A278 (1989) 670.

[8] CHARM II Collaboration, D. Geiregat et al., Nucl. Instrum. and Methods A325 (1993) 92.

[9] V.A. Novikov, L.B. Okun and M.I. Vysotsky, Mod.Phys. Lett. A Brief Reviews 8 (1993) 2529. B302 B

[10] The LEP Collaborations and the LEP Electroweak working group, CERN-PPE/93-157 (1993)

[11] L3 Collaboration, O. Adriani et al., Phys. Lett. B306 (1993) 187.

S. Riemann, private communication.

For an analysis of all LEP data in terms of a different parametrization see also:

G. Altarelli et al., Physics Lett. B318 (1993) 139.

[12] CDF Collaboration, F. Abe et al., Phys. Rev. Lett. 68 (1992) 1463.

[13] J.A. Grifols and S. Peris, Phys. Lett. B168 (1986) 264.

J.L. Rosner, Commun. Nucl Part. Phys.

14 (1985) 229.

[14] CHARM Collaboration, J. Dorenbosch et al., Z. Phys. C41 (1989) 567.

BBKOPS(E734) Collaboration, L.A. Ahrens et al., Phys. Rev. D41 (1990) 3297.

ILM(E225) Collaboration, R.C.Allen et al., Phys. Rev. D47 (1993) 11.
[15] U. Amaldi et al., Phys. Rev. D36 (1987) 1385.

[16] VENUS Collaboration, K. Abe et al., Phys. Lett. B246 (1990) 297.

[17] Particle Data Group, Phys. Rev. D45-II (1992). 